\title{
ESTIMATION OF BETATRON COUPLING AND VERTICAL DISPERSION FOR SPRING-8 STORAGE RING
}

\author{
N. KUMAGAI, H. OHKUMA, K. SOUTOME, M. TAKAO* ${ }^{*}$ H. TANAKA, \\ SPring-8, Mikazuki, Sayo-gun, Hyogo 679-5198, JAPAN
}

\section{Abstract}

The results of investigations on the betatron coupling of the SPring-8 storage ring are described, which imply that the coupling of the normal operation is less than $0.1 \%$. The correction scheme of the vertical dispersion, which limits the vertical emittance as well as the betatron coupling, is also presented.

\section{INTRODUCTION}

The betatron coupling is one of the most important parameters of storage rings for high brilliant light source. By the precise alignment of the magnets [1] and the proper COD correction [2], the SPring-8 storage ring succeeded in achieving the very small linear betatron coupling without skew quadrupole corrector magnets. To prove this fact, we study the betatron coupling of the SPring- 8 storage ring.

The measurement of the vertical emittance of the stored beam is the most preferable method to know the coupling. But, since the vertical emittance of the SPring-8 storage ring is too small to measure, at present we have not yet measured it. Hence, by measuring the dependencies of the various ring parameters on the betatron coupling, we prove the validity of our estimation of the betatron coupling.

\section{COUPLING MEASUREMENTS}

The operation point of the SPring-8 storage ring is in the neighborhood of $(51.16,16.32)$. The difference resonance of $\nu_{x}-\nu_{y} \approx 35$ mainly contributes to the betatron coupling. As changing the horizontal tune while keeping the vertical one constant, We investigate the response of the parameters of the SPring-8 storage ring.

\subsection{Mode Tune Separation}

By the single resonance approximation, the eigen (measured) tunes $\nu_{I}$ and $\nu_{I I}$ are represented by unperturbed tunes $\nu_{x}$ and $\nu_{y}$ as [3]

$$
\nu_{I, I I}=\nu_{x, y} \mp \frac{\Delta}{2} \pm \frac{1}{2} \sqrt{\Delta^{2}+C^{2}},
$$

where $\Delta$ is the distance from resonance and $C$ is the driving term of resonance. Here the integral parts of the tunes are neglected. In Figure 1, we plot the tunes measured while changing operation point. The coupling ratio $\kappa$ is calculated from $\Delta$ and $C$ as

$$
\kappa=\frac{C^{2}}{C^{2}+2 \Delta^{2}},
$$

\footnotetext{
*Email: takao@ spring 8.or.jp
}

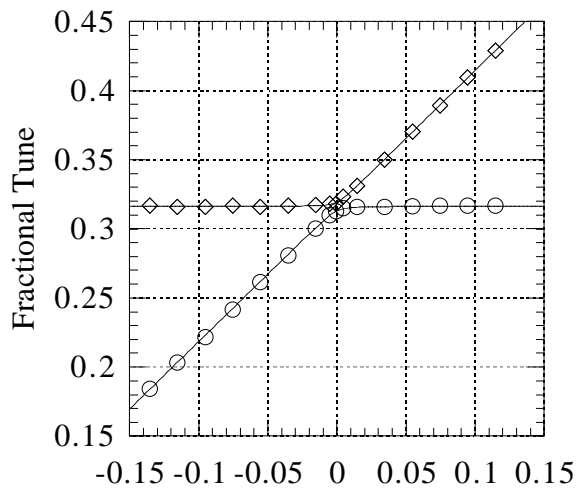

Distance from Resonance $\Delta$

Figure 1: Measurements of mode frequency as a function of detuning.

which is shown in Figure 2. At the normal operation point, the coupling ratio $\kappa$ is estimated to be around $0.06 \%$.

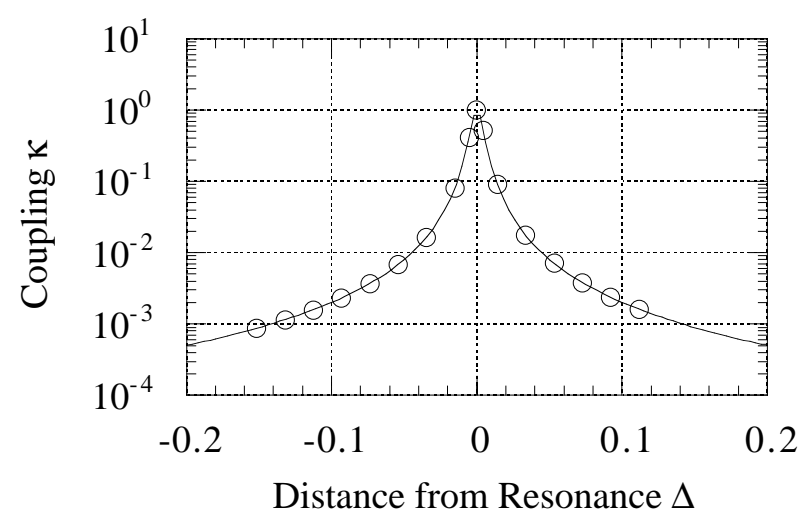

Figure 2: Linear coupling derived from mode tune.

\subsection{Touscheck Lifetime}

The Touscheck effect is crucial for electron storage rings with low emittance since the high beam density increases the probability for scattering between electrons. The Touscheck lifetime is proportional to the bunch volume and hence sensitive to the coupling ratio.

The dependence of the Touscheck lifetime on the coupling ratio is studied, which is shown in Figure 3. The solid line in Figure 3 represents the Touscheck lifetime calculated with the flat beam approximation [5]. The bunch lengths measured at that time did not change significantly in the present range of the coupling ratio, which almost 


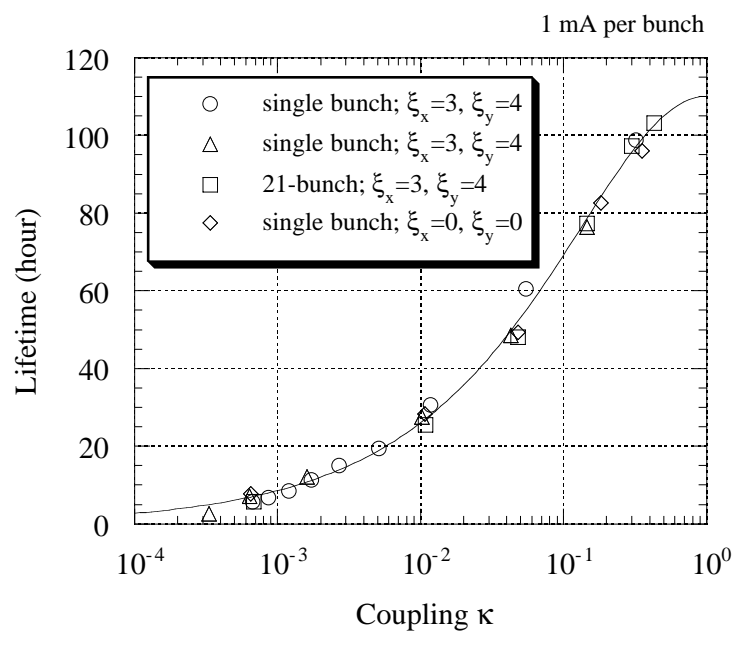

Figure 3: Lifetime as a function of betatron coupling $\kappa$.

equal to $45 \mathrm{ps}$ at single bunch of $1 \mathrm{~mA}$. The dependence of the Touscheck lifetime on the coupling ratio can be fitted to that of the bunch volume

$$
\tau \propto \sigma_{x} \sigma_{y} \sigma_{\ell} \propto \frac{\sqrt{\kappa}}{1+\kappa},
$$

where $\sigma_{x}$ is the bunch width, $\sigma_{y}$ is the bunch height, and $\sigma_{\ell}$ is the bunch length, respectively. The measurements were performed for single bunch with $1 \mathrm{~mA}$ and 21 bunches with $21 \mathrm{~mA}\left(1 \mathrm{~mA}\right.$ per bunch) and chromaticity $\left(\xi_{x}, \xi_{y}\right)=(3$, $4),(0,0)$, which imply that the lifetime is scarcely depend on the chromaticity.

\subsection{Coherent Oscillations}

Although the Touscheck lifetime has the sensitivity to the coupling ratio, there is ambiguity in relating it to the coupling ratio. Hence we more directly study the coupling ratio by measuring the coherent oscillation after horizontal kick in terms of turn-by-turn beam position monitor. One then finds the beats in the envelopes of the coherent oscillations in the horizontal and vertical planes, which originate in the interchange of energy between the two oscillations. By firing a horizontal pulse magnet, we give about $50 \mu \mathrm{rad}$ kick to a single bunch. Measured oscillations with the estimated coupling ratio $0.06 \%, 5 \%$ and $35 \%$ are shown in Figure 4, where the black and gray regions indicate the horizontal and vertical oscillations, respectively.

In terms of the ratio $G$ of the minimum to the maximum of the horizontal envelope oscillation and the period $T$ of the envelope beat oscillations, the distance from resonance $\Delta$ and the coupling driving term $C$ are given by $[3,4]$

$$
\Delta=\frac{G}{T}, \quad C=\frac{\sqrt{1-G^{2}}}{T},
$$

where $T$ is scaled by the revolution period, i.e. equals to the turn number. The coupling ratios estimated from the data of turn-by-turn BPM are given in Figure 5. Note that we
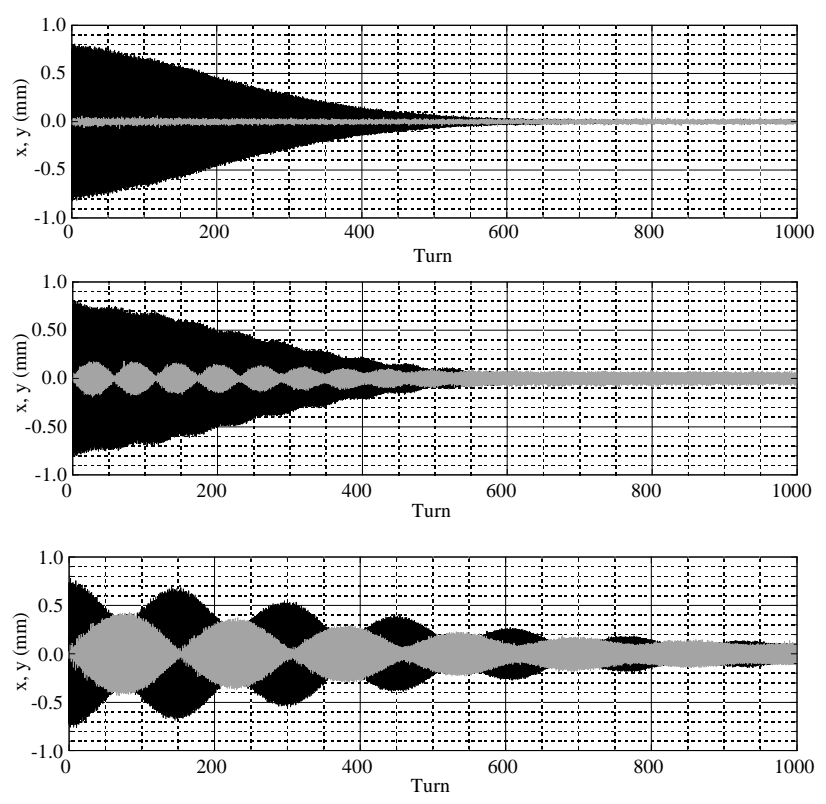

Figure 4: Coherent oscillation measured by turn-by-turn BPM.

can not measure the coupling ratio of the normal operation point by the coherent oscillations. The solid line in Figure 5 corresponds to the coupling ratio calculated from the mode tune separation. Figure 5 shows fairly good agree-

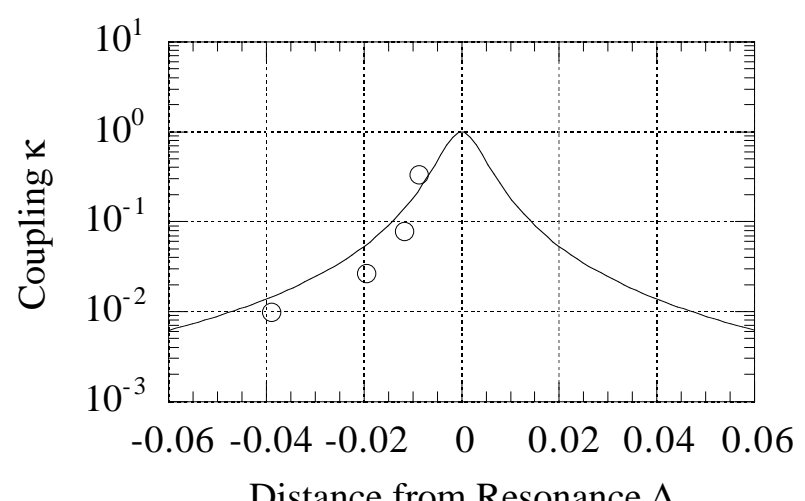

Figure 5: Horizontal Emittance as a function of betatron coupling $\kappa$.

ment between the couplings estimated from the coherent oscillation and derived by mode tune separation.

\subsection{Horizontal Emittance}

The horizontal emittance also directly represents the coupling ratio. By measuring the loss rate of a bunched beam when pushed to septum wall by injection pulse bump, we estimate the bunch width and then the horizontal emittance. Figure 6 shows the loss rate for the cases of the estimated coupling ratio $0.06 \%$ and $43 \%$, where the open circles and the solid line denote the measured coupling and the fitted 
result for the case of coupling $0.06 \%$, and the open triangles and the dashed line for $43 \%$. Figure 7 shows the de-

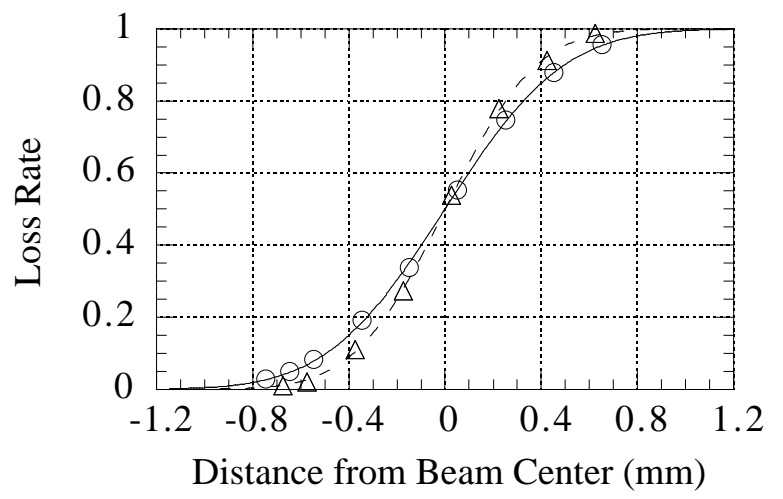

Figure 6: Loss rate of stored current as a function of beam position.

pendence of the horizontal emittance on the coupling ratio, which also implies that we fairly well estimate and control the coupling ratio.

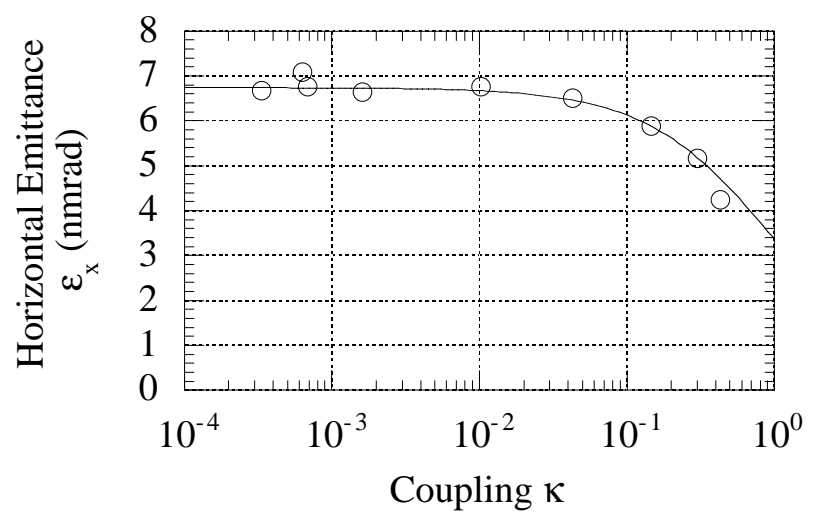

Figure 7: Horizontal Emittance $\epsilon_{x}$ as a function of betatron coupling $\kappa$. The solid line denotes the fitted result of $\epsilon_{x}=\epsilon_{0} /(1+\kappa)$, where $\epsilon_{0}=6.8 \pm 0.7 \mathrm{nmrad}$ is the natural emittance.

\section{CORRECTION}

The coupling ratio of the SPring- 8 storage ring is so small that the faint skew quadrupole fields produced by the gap drives of some insertion devices immediately make the coupling a few times larger. The variation of the lifetime in the operation with several bunches, where the Touscheck effect dominates the beam lifetime, is shown in Figure 8. The sudden jumps of the lifetime correspond to the gap changes of some ID's which generate additional coupling driving term. These extra skew quadrupole fields can be simply corrected by one or two skew quadrupole magnets placed adjacent to the ID's.

At present the spurious vertical dispersion of the SPring8 storage ring is order of one centimeter. The bunch height

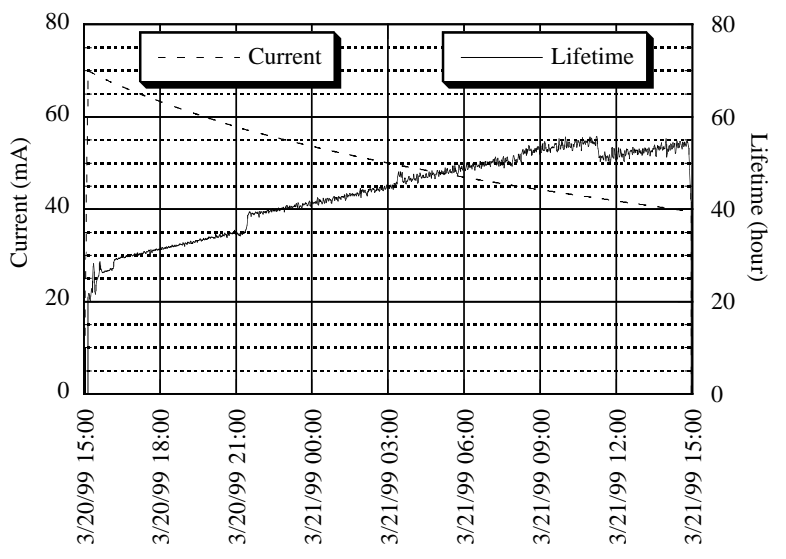

Figure 8: Current and lifetime during the user operation with several bunches.

spread due to the vertical dispersion is comparable to that due to the linear betatron coupling. It is intended to install 24 skew quadrupole magnets at the dispersive sections for correcting the vertical dispersion. The simulation result represented in Figure 9 shows that the skew quadrupole correctors reduce the vertical dispersion by half in r.m.s.. The correction of the vertical dispersion is limited by the measurement accuracy of BPM, which at present is order of a few $\mu \mathrm{m}$ 's. The correction scheme is imposed with the constraint of not exciting any other resonances so that it does not deteriorate the linear betatron coupling.

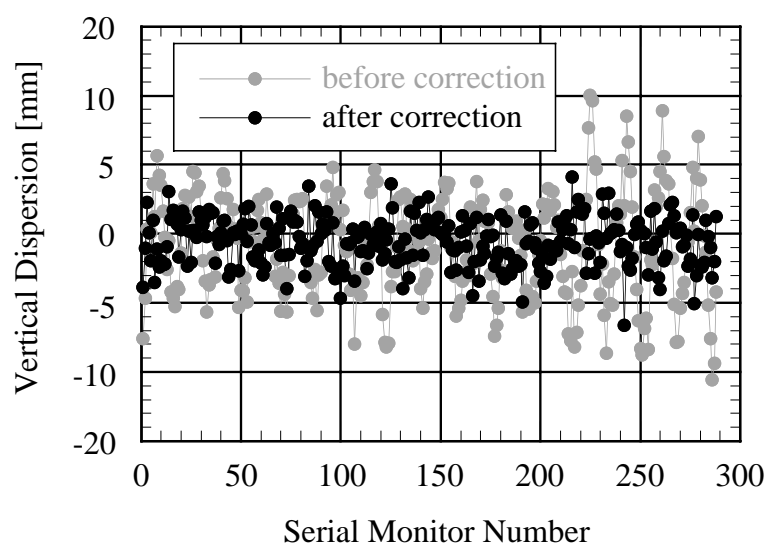

Figure 9: Vertical dispersion before and after correction.

\section{REFERENCES}

[1] H. Tanaka, et al., N.I.M. A313 (1992), 529.

[2] H. Tanaka, et al., SPring-8 Ann. Rep. 1997, p.188; K. Soutome, et al., "Calibration of Beam Position Monitors Using a Stored Beam in the SPring-8 Storage Ring", in these proceedings.

[3] G. Guignard, Phys. Rev. E51 (1995), 6104.

[4] H. Tanaka and A. Ando, Part. Acc. 44 (1994), 17.

[5] C. Bernardini, et al., Phys. Rev. Lett. 10 (1963), 407; J. Le Duff, CERN REP. CERN 89-01 (1989), 114. 